\title{
Serum Level of Matrix Metalloproteinase 3 and Hydroxyproline in Patients with Early Rheumatoid Arthritis
}

\author{
Adel Abbas El Beialy, Hemmat Ahmed Elabd, Asmaa Fouad Ibrahim Abd El-Rahman* \\ Department of Physical Medicine, Rheumatology and Rehabilitation, Faculty of Medicine for Girls, Al- \\ Azhar University, Cairo, Egypt \\ *Corresponding author: Asmaa Fouad Ibrahim Abd El-Rahman, Mobile: (+20) 1113670833 , \\ E-Mail: asmaafouadibrahim@gmail.com
}

\begin{abstract}
Background: Rheumatoid arthritis (RA) is an autoimmune disease that results in a chronic, systemic inflammatory disorder that may affect many tissues and organs, but principally attacks flexible (synovial) joints.

Objective: The aim of this study was to estimate serum level of Matrix metalloproteinase 3 and hydroxyproline in early rheumatoid arthritis.

Patients and methods: This study included a total of 40 patients with early RA (disease duration $<3$ yrs.) and 20 normal volunteers of matched age and sex served as the control group, attending at Department of Physical Medicine, Rheumatology and Rehabilitation, Al-Hussein University hospital. A disease evaluation was done by DAS 28 for all subjects.

Results: In this study, there was a significant positive correlation between matrix metalloproteinase 3 (MMPs) with all studied parameters of disease activity (MS, SJC, GH, ESR, DAS 28) p-value <0.05 and a significant positive correlation between MMP3 and TJC p-value $<0.001$. There was also a significant positive correlation between hydroxyproline with (TJC, SJC, DAS28) p-value <0.05. Results showed a significant positive correlation between matrix metalloproteinase 3 and hydroxyproline. (P-value <0.001).

Conclusion: It could be concluded that in RA there is an evidence of elevated serum MMP3 and HP levels that reflects disease activity and could be used as a specific marker for joint damage.
\end{abstract}

Keywords: Metalloproteinase 3, Hydroxyproline, Rheumatoid arthritis.

\section{INTRODUCTION}

The initial presenting features of early RA do not substantially differ from other inflammatory arthritis. So prior to definite diagnosis, patients with early RA are usually classified as undifferentiated arthritis which difficultly can be discriminated from other inflammatory arthritis. Up to now, early RA was denoted to patients with disease duration of less than 2 years ${ }^{(\mathbf{1})}$.

The onset of disease is not similar in all patients but varies in regard to type, number, and the pattern of joint involvement. The course of disease may be also different according to the presence or absence of several variables including genetic background, frequency of swollen joints, autoantibody in the serum and the severity of inflammatory process ${ }^{(2)}$.

The degradation of articular cartilage and the impairment of joint function are characteristic findings of both Rheumatoid arthritis (RA) and osteoarthritis $(\mathrm{OA})^{(3)}$.

MMPs are a family of zinc-dependent endoproteases with multiple roles in tissue remodeling and degradation of various proteins in the extracellular matrix (ECM). MMPs are commonly classified on the basis of their substrates and the organization of their structural domains into: Collagenases (MMPs-1, -8, and -13), gelatinases (MMPs-2 and -9), stromelysins (MMPs-3, -10, and -11), matrilysins (MMPs-7 and -26), membrane-type metalloproteinases (MT1-6MMPs, also referred to as MMPs-14, $-15,-16,-17$, -24 , and -25), and other MMPs (MMPs-12, -18, $19,-20,-21,-22,-23,-27$, and $-28^{(4)}$.

Matrix metalloproteinases (MMPs) are responsible for the homeostatic tissue repair and degradation of collagens ${ }^{(5)}$.

Previous studies have described the mechanism of collagen degradation by collagenolytic MMPs, which cleave interstitial collagens at a distinct site within their triple-helical region, resulting in 3/4- and 1/4-length fragments. These fragments are thought to readily denature and to be further degraded by the gelatinases, MMP-2, MMP-9, and other proteases ${ }^{(6)}$.

Collagen turnover is crucial during normal physiological processes including wound healing, organogenesis, cell migration, and various inflammatory processes. Overexpression of collagen degrading MMPs results in an excessive ECM degradation that can lead to a variety of pathologies such as rheumatoid arthritis ${ }^{(7)}$.

Mahmoud et al. ${ }^{(8)}$ showed elevation of serum MMP1 in Egyptian patients with RA and OA, elevation of serum MMP3 levels in patients with RA but not in those with $\mathrm{OA}^{(9)}$. 
Hydroxyproline is a nonproteinogenic and nonessential amino acid and it is an important constituent of the major structural protein, collagen and play a key role in the synthesis and stability of the collagen ${ }^{(10)}$.

Collagen, the most abundant protein in mammals, primarily gives rise to the mechanical integrity of connective tissues, such as those of the musculoskeletal system, including bone, cartilage, and tendon. Additionally, other biological structures (e.g., skin, eye, lung, gut, and vasculature) incorporate copious collagen ${ }^{(11)}$.

Hydroxyproline is synthesized by posttranslational hydroxylation of proline during collagen biosynthesis and helps in the sharp twisting of the collagen triple-helix ${ }^{(\mathbf{1 2})}$.

Collagens were considered as the only major resource of hydroxyproline. Recently hydroxyproline is also shown to be present in few other proteins in smaller quantity, e.g., elastin, C1q component of the complement system, acetylcholinesterase, macrophage scavenger proteins, and ectodysplasin $\mathrm{A}^{(\mathbf{1 3})}$.

The colossal amount of hydroxyproline in collagen has made it a valuable measure of collagen and collagen metabolism ${ }^{(14)}$.

Investigations of physiological and pathological collagen metabolism most commonly utilize measurements of hydroxyproline in the plasma, urine and body tissue. Therefore, determination of hydroxyproline provides useful information for the diagnosis and prognosis of diseases caused by disorders of the collagen metabolism ${ }^{(\mathbf{1 5})}$.

The aim of this study was to estimate serum level of Matrix metalloproteinase 3 and hrdroxyproline in early rheumatoid arthritis.

\section{SUBJECTS AND METHODS}

This study included a total of 40 patients with early RA (disease duration $<3$ yrs.) and 20 normal volunteers of matched age and sex served as the control group, attending at Department of Physical Medicine, Rheumatology and Rehabilitation, AlHussein University hospital. Written informed consent from all the subjects were obtained.

\section{Ethical approval:}

Approval of Medical Ethics committee of AlAzhar University was obtained.

The forty patients were 4 males (10\%) and 36 females (90\%). Their ages ranged between 25 to 50 years with mean \pm SD age of $37.98 \pm 8.29$ years and twenty normal volunteers of matched age and sex served as the control group. They were 5 males $(25 \%)$ and 15 females $(75 \%)$ their ages ranged between 30 to 45 years with mean \pm SD age of $37.10 \pm 5.37$ years.

The diagnosis of rheumatoid Arthritis was made according to the American College of Rheumatology (ACR) 2010 criteria $^{(16)}$.

Inclusion criteria: Patients who were diagnosed as early RA:

- With at least 1 joint with definite clinical synovitis.

- Whose synovitis is not better explained by another disease (e.g., lupus, psoriatic arthritis, gout).

- Joint involvement: Consists of swelling or tenderness upon examination.

- At least 1 serology test result is needed for classification.

- At least 1 acute phase reactant test result is needed for classification.

Exclusion criteria: Patients on biological drugs were excluded. Also, patients excluded if they have:

- Osteoporosis.

- Chronic liver failure.

- Chronic renal failure.

- Pregnancy and lactation.

- Current or chronic diarrhea.

- Obstructive or restrictive lung diseases.

- Congestive heart failure.

- Current infections.

- Cigarette smoking.

- Malabsorption of any cause.

- Diabetes mellitus.

- Gout.

\section{All patients were subjected to:}

1- Clinical evaluation: History taking and Full clinical, musculoskeletal examination.

2- Evaluation of disease activity by DAS 28 .

3- Laboratory evaluation in the form of: MMP3, Hydroxy proline, ESR, CRP, RF, CBC, SGOT, SGPT, uric acid, urea, creatinine, plasma glucose level and urine analysis.

History taking including personal history, complaint; taken in the patient's own words, menstrual, gynecological and obstetric history, past history and family history.

Examination including general, systemic examination and locomotor examination:

\section{Evaluation of disease activity by DAS 28:}

DAS stands for 'disease activity score' and the number 28 refers to the 28 joints that are 
examined in this assessment (bilateral shoulders, elbows, wrists, MCPs, PIPS, hips and knees).

DAS28 of greater than 5.1 implies active disease, less than 3.2 low disease activity, and less than 2.6 remission.

DAS $28=0.56 * \operatorname{sqr}(\mathrm{TJC}))+(0.28 *$ $\operatorname{sqr}(\mathrm{SJC}))+(0.7 * \ln (\mathrm{ESR}))+(0.014 * \mathrm{GH})$.

TJC is the number of tender joints of the 28 joint. SJC is the number of swollen joints of the 28 joint. Global health assessed by the patient using a $100 \mathrm{~mm}$ visual analog scale (How do you feel concerning your arthritis? from $0=$ best to $100=$ worst).

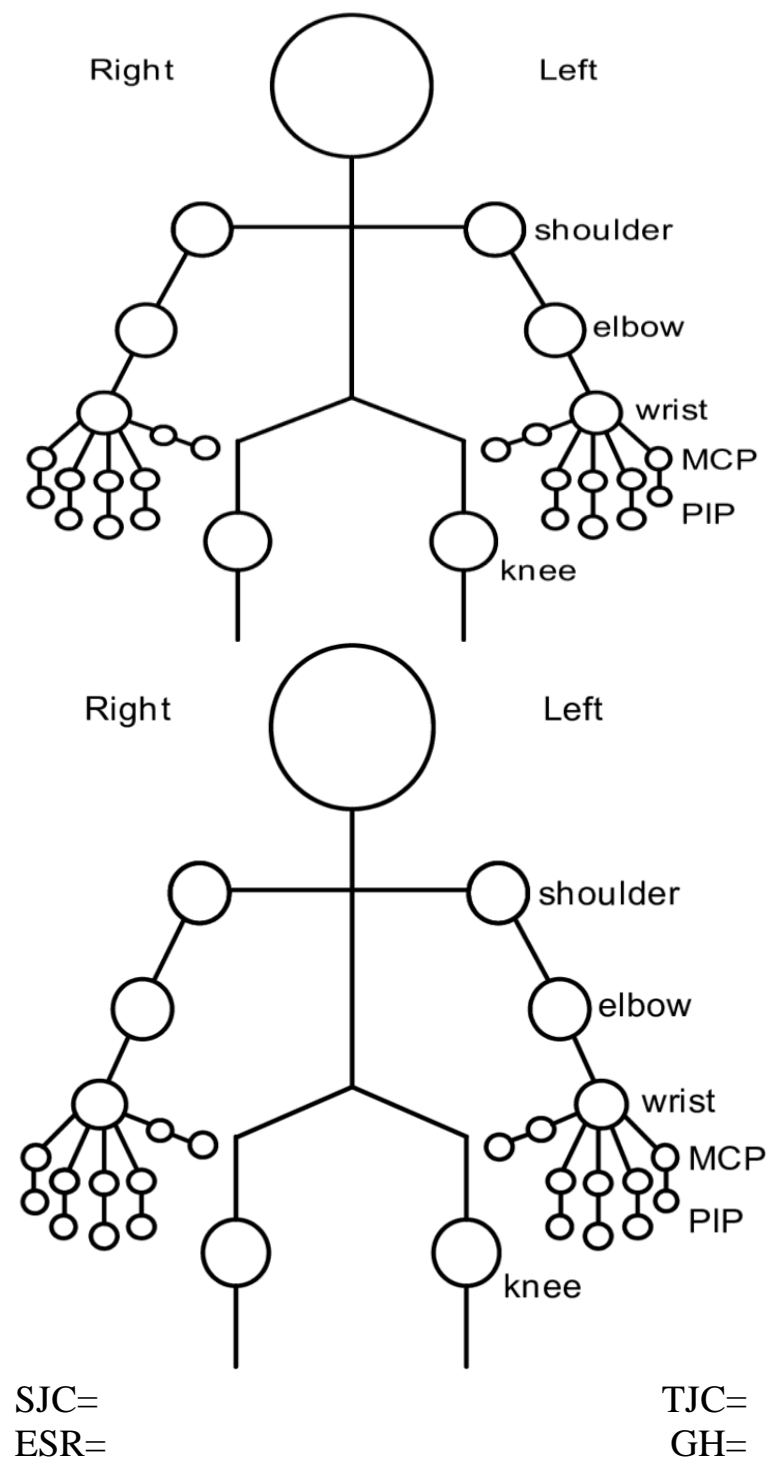

Statistical analysis:

Recorded data were analyzed using the statistical package for social sciences, version 20.0 (SPSS Inc., Chicago, Illinois, USA). Quantitative data were expressed as mean \pm standard deviation (SD). Qualitative data were expressed as frequency and percentage.

\section{The following tests were done:}

- Independent-samples t-test of significance was used when comparing between two means.

- Mann Whitney U test: for two-group comparisons in non-parametric data.

- Chi-square $\left(\mathrm{x}^{2}\right)$ test of significance was used in order to compare proportions between qualitative parameters.

- Pearson's correlation coefficient (r) test was used to assess the degree of association between two sets of variables

- The confidence interval was set to $95 \%$ and the margin of error accepted was set to $5 \%$. So, the p-value was considered significant as the following:

- Probability (P-value)

- P-value $\leq 0.05$ was considered significant.

- P-value $\leq 0.001$ was considered as highly significant.

- P-value >0.05 was considered insignificant.

\section{RESULTS}

This study was conducted on sixty subjects. They were two groups:

- Patient group: It included forty patients diagnosed as early Rheumatoid Arthritis according to the American College of Rheumatology (ACR) 2010 criteria. They were 4 males $(\mathbf{1 0 \%})$ and $\mathbf{3 6}$ females (90\%), their ages ranged between $\mathbf{2 5}$ to $\mathbf{5 0}$ years with mean of $37.98 \pm 8.29$ years.

- Control group: It included twenty subjects of age and sex matched normal volunteers, they had been included to constitute the control group. They were $\mathbf{5}$ males (25\%) and 15 females (75\%), their ages ranged between $\mathbf{3 0}$ to $\mathbf{4 5}$ years with mean of $\mathbf{3 7 . 1 0} \pm \mathbf{5 . 3 7}$ years, as demonstrated in table (1). 
Table (1): Comparison between groups according to demographic data.

\begin{tabular}{|l|l|l|l|l|}
\hline Demographic data & Patients $(\boldsymbol{n}=\mathbf{4 0})$ & Control $(\boldsymbol{n}=\mathbf{2 0})$ & t/x2\# & p-value \\
\hline Age (years) & & & & \\
Mean \pm SD & $37.98 \pm 8.29$ & $37.10 \pm 5.37$ & 0.183 & 0.670 \\
Range & $25-50$ & $30-45$ & & \\
\hline Sex & & & & \\
Male & $4(10.0 \%)$ & $5(25.0 \%)$ & 2.353 & 0.125 \\
Female & $36(90.0 \%)$ & $15(75.0 \%)$ & & \\
\hline
\end{tabular}

Using: $t$-Independent Sample t-test; $\# x^{2}$ : Chi-square test p-value $>0.05 \mathrm{NS}$

This table shows no statistically significant difference between groups according to demographic data.

Comparison between groups according to matrix metalloproteinase 3:

Matrix metalloproteinase 3 in the patient's group is 6.7-689.8 with mean of $153.25 \pm 162.05$. MMP3 in the control group is 20.9-201.1 with mean of 59.79 \pm 63.54 .

When comparing the RA group to the control group, there was statistically significant increase mean of patients group compared to control group according to matrix metalloproteinase $3 p$-value $<0.05 \mathrm{~S}$ as shown in table (2).

Table (2): Comparison between groups according to matrix metalloproteinase3.

\begin{tabular}{|l|l|l|l|l|}
\hline Matrix Metalloproteinase 3 & Patients $(\boldsymbol{n}=\mathbf{4 0})$ & Control $(\boldsymbol{n}=\mathbf{2 0})$ & z-test & p-value \\
\hline Mean \pm SD & $153.25 \pm 162.05$ & $59.79 \pm 63.54$ & \multirow{2}{*}{6.136} & \multirow{2}{*}{$0.016^{*}$} \\
\hline Median (IQR) & $92.5(74.85)$ & $32.15(16.22)$ & & \\
\hline
\end{tabular}

Using: Mann-Whitney test; * -value <0.05 S

\section{Comparison between groups according to hydroxyproline:}

Hydroxyproline ranged in the patients group is $1-24.9$ with mean of $4.81 \pm 6.89$. HP ranged in the control group is 1.4-6.73 with mean of 4.52 \pm 1.55 .

When comparing the RA group to the control group, there was no statistically significant difference between groups according to hydroxyproline $\mathrm{p}$-value $=0.857$ as shown in table (3).

Table (3): Comparison between groups according to hydroxyproline.

\begin{tabular}{|l|l|l|l|l|}
\hline Hydroxyproline & Patients $(\boldsymbol{n}=\mathbf{4 0})$ & Control $(\boldsymbol{n}=\mathbf{2 0})$ & z-test & p-value \\
\hline Mean \pm SD & $4.81 \pm 6.89$ & $4.52 \pm 1.55$ & 0.033 & \multirow{2}{*}{0.857} \\
\hline Median (IQR) & $1.85(3.45)$ & $4.5(2.1)$ & & \\
\hline
\end{tabular}

Using: Mann-Whitney test; *p-value <0.05 S

Correlation between matrix metalloproteinase 3 and hydroxyproline :

There is a Positive significant correlation between matrix metalloproteinase 3 and hydroxyproline with $\mathrm{r}=0.947$ and $\mathrm{p}$-value $<0.001 \mathrm{HS}$ as shown in table (4).

Table (4): Correlation between matrix metalloproteinase 3 and hydroxyproline with demographic data, using Pearson Correlation Coefficient in patients group.

\begin{tabular}{|l|l|l|l|l|}
\hline \multirow{2}{*}{ Patients group } & \multicolumn{2}{|l|}{ Matrix Metalloproteinase 3 } & \multicolumn{2}{l|}{ Hydroxyproline } \\
\cline { 2 - 5 } & $\mathbf{r}$ & p-value & $\mathbf{r}$ & p-value \\
\hline Matrix Metalloproteinase 3 & & & 0.947 & $<0.001^{* *}$ \\
\hline Hydroxyproline & 0.947 & $<0.001^{* *}$ & & \\
\hline Age (years) & -0.063 & 0.701 & -0.205 & 0.203 \\
\hline Disease duration (years) & -0.136 & 0.403 & -0.052 & 0.748 \\
\hline
\end{tabular}

Using: r-Pearson correlation Coefficient

p-value $>0.05 \mathrm{NS} ; * *$-value $<0.001 \mathrm{HS}$

Correlation between matrix metalloproteinase 3 and hydroxyproline with medication:

There is no correlation between matrix metalloproteinase 3 and Hydroxyproline with medication pvalue >0.05 NS as shown in table (5). 
Table (5): Correlation between matrix metalloproteinase 3 and hydroxyproline with medication, using Pearson Correlation Coefficient in patients group.

\begin{tabular}{|c|c|c|c|c|}
\hline \multirow{2}{*}{ Medication } & \multicolumn{2}{|c|}{ Matrix Metalloproteinase 3} & \multicolumn{2}{|c|}{ Hydroxyproline } \\
\hline & $\mathbf{r}$ & p-value & $\mathbf{r}$ & p-value \\
\hline Antimalarial & -0.159 & 0.328 & -0.215 & 0.182 \\
\hline MTX & 0.106 & 0.517 & -0.011 & 0.946 \\
\hline Leflunamide & 0.208 & 0.198 & 0.191 & 0.237 \\
\hline Prednisone & -0.144 & 0.374 & -0.154 & 0.344 \\
\hline
\end{tabular}

Using: r-Pearson correlation Coefficient p-value $>0.05 \mathrm{NS}$

Correlation between matrix metalloproteinase 3 and hydroxyproline with parameters of disease activity:

There is a Positive significant correlation between matrix metalloproteinase 3 and MS, SJC, GH, ESR, DAS28 $p$ value <0.05 S, also Positive significant correlation between MMP3 and TJC p-value <0.001 HS as shown in table(6).

There is a Positive significant correlation between Hydroxyproline with all studied parameters of disease activity p-value $<0.05 \mathrm{~S}$ as shown in table (6) and.

Table (6): Correlation between matrix metalloproteinase 3 and hydroxyproline with parameters of disease activity, using Pearson Correlation Coefficient in patients group.

\begin{tabular}{|c|c|c|c|c|}
\hline \multirow{2}{*}{ Parameters of disease activity } & \multicolumn{2}{|c|}{ Matrix Metalloproteinase 3} & \multicolumn{2}{|c|}{ Hydroxyproline } \\
\hline & $\mathbf{r}$ & p-value & $\mathbf{r}$ & p-value \\
\hline MS & 0.480 & $0.002 *$ & 0.274 & $0.046^{*}$ \\
\hline TJC & 0.497 & $<0.001 * *$ & 0.357 & $0.024 *$ \\
\hline SJC & 0.463 & $0.003^{*}$ & 0.343 & $0.030 *$ \\
\hline $\mathrm{GH}$ & 0.380 & $0.015^{*}$ & 0.275 & $0.048 *$ \\
\hline ESR & 0.361 & $0.022 *$ & 0.293 & $0.039 *$ \\
\hline DAS 28 & 0.446 & $0.004 *$ & 0.311 & $0.029 *$ \\
\hline
\end{tabular}

Using: r-Pearson correlation Coefficient

p-value >0.05 NS; *p-value <0.05 $\mathrm{S} ;{ }^{* *} p$-value $<0.001 \mathrm{HS}$

\section{DISCUSSION}

The aim of this study was to estimate serum level of matrix metalloproteinase 3 and hydroxyproline in early rheumatoid arthritis and correlate it with the disease activity. To elucidate this role forty Egyptian early rheumatoid arthritis patients and twenty age and sex matched healthy control subjects were included in this study.

Regarding the RA patients, they were 4 males (10\%) and 36 females (90\%), their ages ranged between 25 to 50 years with mean \pm SD age of $37.98 \pm 8.29$ years.

As regard to duration of the disease, in the current study the disease duration ranged from 0.08 to 3 years with the mean \pm SD of $1.54 \pm 1.12$ years.

Regarding the control group, they were $\mathbf{5}$ males (25\%) and 15 females (75\%) their ages ranged between 30 to $\mathbf{4 5}$ years with mean \pm SD age of $\mathbf{3 7 . 1 0} \pm \mathbf{5 . 3 7}$ years.
Regarding the RA patients, the diagnosis of rheumatoid Arthritis was made according to the American College of Rheumatology (ACR) 2010 criteria, also the patients were selected after applying the inclusion and the exclusion criteria.

All patients were subjected to laboratory evaluation in the form of MMP3, Hydroxy proline, ESR, CRP, RF, CBC, SGOT, SGPT, uric acid, urea, creatinine, plasma glucose level and urine analysis.

\section{- Evaluation of disease activity by DAS} 28.

In this study serum MMP3 was significantly higher in RA patients than controls p-value $<0.05$, which agrees with Harvey et al. ${ }^{(17)}$.

Posthumus et al. ${ }^{(18)}$ study showed that MMP3 levels in control group were low or undetectable 
$(\mathrm{p}=0.19)$ and levels in RA group were higher than control group $(\mathrm{p}<0.01)$.

Mahmoud et al. ${ }^{(8)}$ showed elevation of serum MMP1 in Egyptian patients with RA and OA and elevation of serum MMP3 levels in patients with RA.

Tchetverikov et al. ${ }^{(19)}$ study showed that proMMP-3, -8, and -9 levels were increased in the serum of patients with RA when compared with the control group.

Zhou et al. (20) Who collected serum samples from 151 patients with RA and 43 healthy control, the result shows that The serum MMP-3 levels in all patients with RA were significantly higher than those in the healthy controls ( $\mathrm{p}<$ $0.001)$

In this study, there was a significant positive correlation between matrix metalloproteinase 3 with all studied parameters of disease activity (MS, SJC, GH, ESR, DAS 28) p-value <0.05 S, also significant positive correlation between MMP3 and TJC p-value $<0.001 \mathrm{HS}$.

This agrees with the result of So et al. ${ }^{(21)}$, Posthumus et al. ${ }^{(18)}$ and Shinozaki et al. ${ }^{(22)}$ who found that the serum concentrations of MMP-3 correlate with disease activity and predict the progression of joint destruction in RA.

Zhou et al. ${ }^{(20)}$ who found that the ESR, DAS28, and HAQ in each group of patients with active RA were significantly higher than those in patients with stable RA ( $p<0.01$ or $\mathrm{p}<0.001)$.

They suggested that serum MMP3 is a marker of systemic inflammation in RA and it may therefore be a useful measurable marker of active RA.

However, Abd-Allah et al. ${ }^{\text {(23) }}$ showed that there were no significant associations between MMP1 and MMP3 levels and duration of the diseases, activity.

Our study showed a significant positive correlation between matrix metalloproteinase 3 with ESR,CRP p-value <0.05 $\mathrm{S}$, which comes in agreement with the results of So et al. ${ }^{(21)}$, which showed a strong positive correlation between serum MMP3 and $\mathrm{CRP}(\mathrm{p}<0.008)$, and also $\operatorname{ESR}(\mathrm{p}<0.006)$.

Posthumus et al. (18) found significant correlation between serum MMP3 and CRP.

Since the production of acute phase proteins like CRP, as well as the production of MMP3, is stimulated by pro-inflammatory cytokines like IL-1 and TNF- $\alpha$, these correlation could be expected.

Tchetverikov et al. (19) found that proMMP-3 levels in SF correlated with CRP levels.

Also, Peak et al. ${ }^{(24)}$ who collected the serum of 82 RA patients, showed significant correlation bet serum MMP3 and ESR ( $p=0.01)$.

In this study, there was no correlation between matrix metalloproteinase with medication p-value $>0.05 \mathrm{NS}$.

This study showed no statistically significant difference between the early RA patients and the control groups according to hydroxyproline) $\mathrm{p}$-value $<0.05 \mathrm{~S}$ ).

This agrees with the result of Ahmed $\boldsymbol{e t}$ al. (25) who collected 16 healthy control, 16 patients with early OA (eOA), 10 patients with early RA (eRA) and 10 patients with arthritic disease other than RA (non RA ).he found that Plasma hydroxyproline was increased $44 \%$ in eOA and $58 \%$ in non-RA but not increased in eRA with respect to healthy controls. So, it was important in discriminating disease versus control but not for typing of early-stage arthritis ${ }^{(25)}$.

Also this agrees with the result of Sambrook et al. ${ }^{(26)}$ who found that mean urinary hydroxyproline-creatinine ratios were increased in RA, but these differences were not significant compared with control values. But several patient values were markedly increased.

In our study, there was a significant Positive correlation between Hydroxyproline with (TJC, SJC, DAS28) p-value <0.05 S. Which agrees with Sambrook et al. (26) who found that urinary hydroxyproline: creatinine ratios correlated with joint count $(\mathrm{r}=0.62, \mathrm{p}<0.01)$.

In our study, there was a significant positive correlation between Hydroxyproline with ESR pvalue $<0.05 \mathrm{~S}$. which agrees with Sambrook et al. (26) who found that urinary hydroxyproline: creatinine ratios correlated positively with ESR $(\mathrm{r}=0.70, \mathrm{p}<0.01)$.

In our study, there was an insignificant correlation between Hydroxyproline with CRP pvalue $>0.05$ NS. However, Sambrook et al. (26) found a positive correlation between urinary hydroxyproline: creatinine ratios CRP ( $\mathrm{r}=0.70$, $\mathrm{p}<001)$.

Our study showed a significant positive correlation between matrix metalloproteinase 3 and hydroxyproline. (P-value <0.001 HS). However, Tchetverikov et al. (19) found that SF Hydroxyproline levels did not correlate with proMMP-3. 
In this study, No correlation was found between Hydroxyproline and medication p-value $>0.05 \mathrm{NS}$.

Early-stage detection of arthritic disease and effective lifestyle and pharmaceutical interventions could achieve a cure for RA and improve outcomes for other arthritic disease ${ }^{(27)}$.

Development of drugs to suppress oxidative damage and glycation damage to collagen in arthritis will be facilitated by availability of companion diagnostic tests based on related biomarkers ${ }^{\mathbf{2 8}}$.

In addition to understanding the pathogenesis, pathophysiology and treatment strategies of several diseases, using hydroxyproline as a biomarker may provide useful information for both biomedical research and drug development (14).

This study shows that high serum level of MMP3 reflects high disease activity in RA patients and its measurement may be a useful marker for more destructive joint disease in RA and suppression of MMP3 production may be an effective therapeutic approach for patients with early RA.

\section{CONCLUSION}

It could be concluded that:

- High serum level of MMP3 reflects high disease activity in RA patients and its measurement may be a useful marker for more destructive joint disease in RA. It is expected that suppression of MMP3 production may be an effective therapeutic approach for patients with early RA.

- High serum level of HP reflects high disease activity in RA patients and its measurement may be a useful marker for the prognosis (degree of destruction through the joints).

- There is a significant positive correlation between matrix metalloproteinase 3 and hydroxyproline.

- Serum levels of MMP3 and HP may be a useful marker for the prognosis because it will give us an idea about the degree of destruction through the joints.

\section{RECOMMENDATIONS}

- Seeking medical advice as early as possible is a must for any patient complains of symptoms of arthritis specially hand arthritis for early diagnosis and the best control of disease.

- MMP3 measurement for RA patients because it is a useful marker for expecting the destructive course of the disease.
- HP measurement for RA patients because it is a useful marker for expecting the instant destruction throught the joints.

\section{REFERENCES}

1. Scott DL (2007): Early rheumatoid arthritis. Br. Med. Bull., 81-82, 97-114.

2. Gossec L, Combescure C, Rincheval $\mathbf{N}$ et al. (2010): Relative clinical influence of clinical, laboratory, and radiological investigations in early arthritis on the diagnosis of rheumatoid arthritis. Data from the French Early Arthritis Cohort ESPOIR. J. Rheumatol., 37(12): 2486-2492.

3. Yoshihara Y, Nakamura H, Obata $\mathrm{K}$ et al. (2000): Matrix metalloproteinases and tissue inhibitors of metalloproteinases in synovial fluids from patients with rheumatoid arthritis or osteoarthritis. Ann. Rheum. Dis., 59(6): 455-461.

4. Paiva KB, Granjeiro JM (2014): Bone tissue remodeling and development: focus on matrix metalloproteinase functions. Arch. Biochem. Biophys., 561: 74-87.

5. Bonnans C, Chou J, Werb $Z$ (2014): Remodelling the extracellular matrix in development and disease. Nat. Rev. Mol. Cell Biol., 15(12): 786-801.

6. Toth M, Sohail A, Fridman $R$ (2012): Assessment of gelatinases (MMP-2 and MMP-9) by gelatin zymography. Methods Mol. Biol., 878: 121-135.

7. Rohani MG, McMahan RS, Razumova MV et al. (2015): MMP-10 Regulates Collagenolytic Activity of Alternatively Activated Resident Macrophages. J. Invest. Dermatol., 135(10): 23772384.

8. Mahmoud RK, El-Ansary AK, El-Eishi HH et al. (2005): Matrix metalloproteinases MMP-3 and MMP-1 levels in sera and synovial fluids in patients with rheumatoid arthritis and osteoarthritis. Ital. J. Biochem., 54(3-4): 248-257.

9. Cowell S, Knauper V, Stewart ML et al. (1998): Induction of matrix metalloproteinase activation cascades based on membrane-type 1 matrix metalloproteinase: associated activation of gelatinase A, gelatinase B and collagenase 3 . Biochem. J., 331 (2): 453-458.

10. Paul S (2011): Fish bone chemistry and ultrastructure: implications for taphonomy and stable isotope analysis. J. Archaeol. Sci., 38(12): 3358-3372.

11. Nair AK, Gautieri A, Buehler MJ (2014): Role of intrafibrillar collagen mineralization in defining the compressive properties of nascent bone. Biomacromolecules, 15(7): 2494-2500.

12. Kadler KE, Hill A, Canty-Laird EG (2008): Collagen fibrillogenesis: fibronectin, integrins, and minor collagens as organizers and nucleators. Curr. Opin. Cell Biol., 20(5): 495-501.

13. Bochicchio B, Laurita A, Heinz A et al. (2013): Investigating the role of (2S,4R)-4-hydroxyproline in elastin model peptides. Biomacromolecules, 14(12): 4278-4288. 
14. Srivastava AK, Khare $P$, Nagar HK et al. (2016): Hydroxyproline: A Potential Biochemical Marker and Its Role in the Pathogenesis of Different Diseases. Curr. Protein Pept. Sci., 17(6): 596-602.

15. Hofman K, Hall B, Cleaver H et al. (2011): Highthroughput quantification of hydroxyproline for determination of collagen. Anal. Biochem., 417(2): 289-291.

16. Aletaha D, Neogi T, Silman AJ et al. (2010): 2010 Rheumatoid arthritis classification criteria: an American College of Rheumatology/European League Against Rheumatism collaborative initiative. Arthritis. Rheum., 62(9): 2569-2581.

17. Harvey RA, Ferrier DR (1994): Lippincott's Illustrated Reviews: Biochemistry, 2nd ed.; Lippincott Williams \& Wilkins: Philadelphia, Pp. 43-47.

18. Posthumus MD, Limburg PC, Westra J et al. (2003): Serum matrix metalloproteinase 3 levels in comparison to $\mathrm{C}$-reactive protein in periods with and without progression of radiological damage in patients with early rheumatoid arthritis. Clin. Exp. Rheumatol., 21(4): 465-472.

19. Tchetverikov I, Ronday HK, Van EB et al. (2004): MMP profile in paired serum and synovial fluid samples of patients with rheumatoid arthritis. Ann. Rheum. Dis., 63(7): 881-883.

20. Zhou L, Wang G, Liu $X$ et al. (2017): Matrix metalloproteinase-3 and the 7-joint ultrasound score in the assessment of disease activity and therapeutic efficacy in patients with moderate to severe rheumatoid arthritis. Arthritis Res. Ther., 19(1): 250.

21. So A, Chamot AM, Peclat V et al. (1999): Serum MMP-3 in rheumatoid arthritis: correlation with systemic inflammation but not with erosive status. Rheumatology. (Oxford), 38(5): 407-410.

22. Shinozaki M, Inoue E, Nakajima A et al. (2007): Elevation of serum matrix metalloproteinase-3 as a predictive marker for the long-term disability of rheumatoid arthritis patients in a prospective observational cohort IORRA. Mod. Rheumatol., 17(5): 403-408.

23. Abd-Allah SH, Shalaby SM, Pasha HF et al. (2012): Variation of matrix metalloproteinase 1 and 3 haplotypes and their serum levels in patients with rheumatoid arthritis and osteoarthritis. Genet. Test. Mol. Biomarkers, 16(1): 15-20.

24. Peake NJ, Khawaja K, Myers A et al. (2005): Levels of matrix metalloproteinase (MMP)-1 in paired sera and synovial fluids of juvenile idiopathic arthritis patients: relationship to inflammatory activity, MMP-3 and tissue inhibitor of metalloproteinases-1 in a longitudinal study. Rheumatology, 44(11): 1383-1389.

25. Ahmed U, Anwar A, Savage RS et al. (2015): Biomarkers of early stage osteoarthritis, rheumatoid arthritis and musculoskeletal health. Sci. Rep., 5: 9259.

26. Sambrook PN, Ansell BM, Foster S et al. (1985): Bone turnover in early rheumatoid arthritis. 2. Longitudinal bone density studies. Ann. Rheum. Dis., 44(9): 580-584.

27. Roos EM, Arden NK (2016): Strategies for the prevention of knee osteoarthritis. Nat. Rev. Rheumatol., 12(2): 92-101.

28. Gibson DS, Bustard MJ, McGeough CM et al. (2015): Current and future trends in biomarker discovery and development of companion diagnostics for arthritis. Expert. Rev. Mol. Diagn., 15(2):219-234. 